\title{
European Options under Proportional Transaction Costs: An Algorithmic Approach to Pricing and Hedging
}

\author{
Alet Roux ${ }^{1)}$, Krzysztof Tokarz ${ }^{2)}$ and Tomasz Zastawniak ${ }^{3)}$ \\ 1) Department of Mathematics, University of York \\ Heslington, York YO10 5DD, United Kingdom \\ ar521@york.ac.uk \\ ${ }^{2)}$ Graduate School of Business, National-Louis University \\ ul. Zielona 27, 33-300 Nowy Sacz, Poland \\ ktokarz@wsb-nlu.edu.pl \\ ${ }^{3)}$ Department of Mathematics, University of York \\ Heslington, York YO10 5DD, United Kingdom \\ tz506@york.ac.uk
}

\begin{abstract}
The paper is devoted to optimal superreplication of European options in the discrete setting under proportional transaction costs on the underlying asset. In particular, general pricing and hedging algorithms are developed. This extends previous work by many authors, which has been focused on the binomial tree model and options with specific payoffs such as calls or puts, often under certain bounds on the magnitude of transaction costs. All such restrictions are hereby removed. The results apply to options with arbitrary payoffs in the general discrete market model with arbitrary proportional transaction costs. Numerical examples are presented to illustrate the results and their relationships to the earlier work on pricing options under transaction costs.
\end{abstract}

\section{Introduction}

The question of pricing and hedging of European options in the presence of proportional transaction costs has been examined by many authors. The first to consider the problem was Merton [Mer89], Mer90, followed by Dermody and Rockafellar DR91, Boyle and Vorst BV92, Bensaid, Lesne, Pagès and Scheinkman [BLPS92, Edirisinghe, Naik and Uppal [ENU93, Jouini and Kallal [JK95, Kusuoka Kus95, Koehl, Pham and Touzi [KPT96, KPT99, KPT01, 
Stettner Ste97, Ste00, Perrakis and Lefoll [PL97, Rutkowski Rut98, Touzi Tou99, Jouini Jou00, Palmer Pal01b, Pal01a, Kociński Koc04, Chen, Palmer and Sheu CPS05, and others.

The above papers are concerned with the upper and lower hedging prices that constitute the bounds of the no-arbitrage interval of option prices under transaction costs. These prices will also be referred to as the ask and bid option prices, see Definition 3.1. The significance of the ask and bid option prices is not only in determining the no-arbitrage interval, but also in that they are the liquidity prices. Namely, the option can be bought or sold on demand at the ask price or, respectively, at the bid price. Liquidity is important, in particular, when an option is used as part of a strategy to hedge another derivative security, as is often the case in practice.

In contrast, preference-based approaches lead to intermediate option prices within the no-arbitrage interval. They include pricing based on expected utility maximisation, see Hodges and Neuberger [HN89, Mercurio and Vorst MV97, Constantinides and Perrakis CP02, Davis, Panas and Zariphopoulou DPZ93, Constantinides and Zariphopoulou [CZ99, CZ01, Monoyios [Mon03, Mon04, as well as risk minimisation as in Mercurio and Vorst [MV97 or Lamberton, Pham and Schweizer LPS98.

The present work follows the former group of papers in studying the ask and bid option prices that determine the no-arbitrage interval. The main new contribution is an algorithm for computing the ask and bid prices of options with arbitrary payoffs in an arbitrary discrete model under proportional transaction costs of any magnitude. This significantly extends the techniques in the earlier papers. Another important contribution is an optimal hedging algorithm, which also applies to arbitrary option payoffs and arbitrary discrete models under proportional transaction costs of any magnitude.

To explain the idea behind the pricing algorithm, let us consider the concave function $x \mapsto Z_{0}^{x}$ such that

$$
Z_{0}^{x}=\inf (\alpha+x \beta)
$$

for each $x \in \mathbb{R}$, where the infimum is taken over all portfolios $(\alpha, \beta)$ of cash and stock held at time 0 that are sufficient to hedge the option writer's position, in the sense that there is a self-financing strategy starting with $(\alpha, \beta)$ that superreplicates the option payoff. In the discrete setting the infimum can in fact be replaced by a minimum for any $x$ such that the infimum is finite, and $Z_{0}$ turns out to be a polyhedral (piecewise linear) concave function. A portfolio $(\alpha, \beta)$ held at time 0 is sufficient to hedge the option writer's position if and only if for each $x \in \mathbb{R}$

$$
\alpha+x \beta \geq Z_{0}^{x} .
$$

Pricing the option can therefore be reduced to computing the polyhedral concave function $Z_{0}$. Namely, to be able to hedge his/her position, a writer without any initial stock holdings would need to receive a cash amount $\alpha$ such that $\alpha \geq Z_{0}^{x}$ for each $x \in \mathbb{R}$. The smallest such amount

$$
\alpha=\max _{x \in \mathbb{R}} Z_{0}^{x}
$$


will be the ask price of the option.

Algorithm 4.1 is essentially a dynamic programming procedure on the tree representing the discrete stock price model. It proceeds by backward induction to compute a polyhedral concave function $Z_{t}(\mu)$ with similar properties to those mentioned above for each time $t$ node $\mu$ of the tree, starting with the nodes at the expiry time $T$ of the option. The function $Z_{0}$ is thus obtained at the root node of the tree, and the option price computed as the maximum of $Z_{0}$. The convex optimisation problem for $Z_{0}$ is thereby reduced to a series of much simpler local optimisation problems at each node, resulting in an efficient and general algorithm.

Algorithm 4.2 for computing the writer's hedging strategy is closely related to the above and based on the following property, which will be formalised in Lemma 4.1: If a portfolio $(\gamma, \delta)$ satisfying $\gamma+x \delta \geq Z_{t}^{x}(\mu)$ for each $x \in \mathbb{R}$ is held at a node $\mu$ of the tree, then a new portfolio $(\rho, \sigma)$ can be constructed from $(\gamma, \delta)$ in a self-financing manner such that $\rho+x \sigma \geq Z_{t+1}^{x}(\nu)$ for each $x \in \mathbb{R}$ and each successor node $\nu$ of $\mu$. This makes it possible to string such portfolios together to construct a self-financing strategy hedging the option writer's position.

Thanks to a simple transformation between long and short positions in a European option, the same algorithms can be used to compute the bid price and to hedge the option buyer's position.

In Theorem 4.2 we establish the correctness of both algorithms and, as a byproduct, re-prove (by a very different method) a version of Joinini and Kallal's JK95 representation of the ask option price in terms of the optimal risk-neutral probability measure under proportional transaction costs.

Three numerical examples are presented. The first two are based on the results computed by Boyle and Vorst BV92, Edirisinghe, Naik and Uppal [ENU93] and Palmer Pal01a] for the binomial tree model. The examples serve to show that the new algorithm extends all such approaches to a much wider class of options and parameter ranges in a general and efficient manner. The third example involves a trinomial tree and a basket option to illustrate the claim that the method is capable of dealing with arbitrary discrete models and arbitrary option payoffs.

Option pricing procedures based on minimising the cost of supperreplication, such as those in BLPS92 or ENU93, or maximising the risk-neutral expectation of the payoff, as in the representations in JK95 or Jou00, grow exponentially as the trading frequency increases. This is because the optimal superreplicating strategy and the optimal risk-neutral probability measure that give the option price are, in general, path-dependent, even for options with path-independent payoff. Meanwhile, the polyhedral concave functions $Z_{t}(\mu)$ in Algorithm 4.1 are path-independent, resulting in only polynomial growth. This accounts for the efficiency of the pricing algorithm. 


\section{Model Specifications and Notation}

We consider asset models with discrete time $t=0,1, \ldots, T$ and finite probability space $\Omega$ equipped with a probability measure $Q$ on the sigma-field $\mathcal{F}$ of all subsets of $\Omega$ such that $Q\{\omega\}>0$ for each $\omega \in \Omega$. In addition, a filtration $\{\emptyset, \Omega\}=\mathcal{F}_{0} \subset \mathcal{F}_{1} \subset \cdots \subset \mathcal{F}_{T}=\mathcal{F}$ is given. For any $t=0,1, \ldots, T$ we denote by $\Omega_{t}$ the set of atoms of $\mathcal{F}_{t}$, and identify $\mathcal{F}_{t}$-measurable random variables with functions defined on $\Omega_{t}$.

The filtration can be thought of in terms of a tree, the atoms of $\mathcal{F}_{t}$ being identified with the nodes of the tree at time $t$. For nodes $\mu \in \Omega_{t}$ and $\nu \in \Omega_{t+1}$ such that $\nu \subset \mu$ we say that $\nu$ is a successor node of $\mu$. We denote by

$$
\operatorname{succ} \mu=\left\{\nu \in \Omega_{t+1} \mid \nu \subset \mu\right\}
$$

the set of successor nodes of $\mu \in \Omega_{t}$.

The market model will consist of a risk-free and a risky security, a bond (or cash) and a stock. Trading in stock is subject to proportional transaction costs: at any time $t=0, \ldots, T$ a share can be bought for the ask price $S_{t}^{\text {a }}$ or sold for the bid price $S_{t}^{\mathrm{b}}$, where $S_{t}^{\mathrm{a}} \geq S_{t}^{\mathrm{b}}>0$. The processes $S^{\mathrm{a}}$ and $S^{\mathrm{b}}$ are adapted to the filtration.

Without loss of generality, we assume the bond to be a risk-free security with zero interest rate, the bond price being 1 for all $t=0,1, \ldots, T$. Equivalently, all prices can be regarded as discounted prices.

\subsection{Self-Financing Strategies, Arbitrage and Risk-Neutral Measures}

We define the time $t=0, \ldots, T$ liquidation value $\vartheta_{t}$ of a portfolio $(\xi, \zeta)$ of cash and stock as

$$
\vartheta_{t}(\xi, \zeta)=\xi+\zeta^{+} S_{t}^{\mathrm{b}}-\zeta^{-} S_{t}^{\mathrm{a}}
$$

Definition 2.1 A self-financing strategy is a pair $(\alpha, \beta)$ of predictable processes $\alpha_{t}, \beta_{t}$ representing positions in cash and stock at $t=0, \ldots, T$ such that $\beta_{0}=0$ and

$$
\vartheta_{t}\left(\alpha_{t}-\alpha_{t+1}, \beta_{t}-\beta_{t+1}\right) \geq 0
$$

for each $t=0, \ldots, T-1$. The set of such strategies will be denoted by $\Phi\left(S^{\mathrm{a}}, S^{\mathrm{b}}\right)$.

Definition 2.2 An arbitrage opportunity is a strategy $(\alpha, \beta) \in \Phi\left(S^{\mathrm{a}}, S^{\mathrm{b}}\right)$ such that $\alpha_{0} \leq 0, \vartheta_{T}\left(\alpha_{T}, \beta_{T}\right) \geq 0$ and $Q\left\{\vartheta_{T}\left(\alpha_{T}, \beta_{T}\right)>0\right\}>0$.

Definition 2.3 We say that $P$ is a risk-neutral probability if $P$ is a probability measure on $\Omega$ equivalent to $Q$ and there is a martingale $S$ under $P$ such that $S_{t}^{\mathrm{b}} \leq S_{t} \leq S_{t}^{\mathrm{a}}$ for each $t=0, \ldots, T$.

In what follows we shall denote by $\mathcal{P}$ the set of pairs $(P, S)$ such that $P$ and $S$ satisfy the conditions in Definition 2.3. We shall also use the larger set $\overline{\mathcal{P}}$ of pairs $(P, S)$ such that $P$ is a probability measure on $\Omega$ (not necessarily 
equivalent to $Q$ ) and $S$ is a martingale under $P$ that satisfies $S_{t}^{\mathrm{b}} \leq S_{t} \leq S_{t}^{\mathrm{a}}$ for each $t=0, \ldots, T$.

The following result, obtained by Jouini and Kallal [JK95, who used a slightly different notion of arbitrage, referred to as 'free lunch' in their work, is also valid under the above definition of an arbitrage opportunity, as shown in Tokarz Tok04. See also Ortu Ort01.

Theorem 2.1 (Jouini and Kallal [JK95]) There is no arbitrage opportunity if and only if $\mathcal{P} \neq \emptyset$.

\subsection{Concave Functions}

Here we collect some information about concave functions which will be used in this paper, referring to Rockafellar [Roc97] for more details.

By a proper concave function we mean any function $f: \mathbb{R} \rightarrow \mathbb{R} \cup\{-\infty\}$ such that for any $x \leq y \leq z$

$$
(z-x) f(y) \geq(z-y) f(x)+(y-x) f(z)
$$

and $f(x)>-\infty$ for some $x \in \mathbb{R}$. The effective domain of such a function $f$ is defined as

$$
\operatorname{dom} f=\{x \in \mathbb{R} \mid f(x)>-\infty\} .
$$

Moreover, we say that $f$ is a polyhedral proper concave function if $\operatorname{dom} f$ is closed and there exist real numbers $a_{1}, \ldots, a_{n}$ and $b_{1}, \ldots, b_{n}$ such that for each $x \in \operatorname{dom} f$

$$
f(x)=\min _{i=1, \ldots, n}\left(a_{i} x+b_{i}\right) .
$$

Definition 2.4 The concave cap $\operatorname{cap}\left\{f_{1}, \ldots, f_{n}\right\}$ of functions $f_{1}, \ldots, f_{n}$ : $\mathbb{R} \rightarrow \mathbb{R} \cup\{-\infty\}$ is defined as the smallest concave function $h$ such that

$$
h \geq \max \left\{f_{1}, \ldots, f_{n}\right\} .
$$

Lemma 2.2 Suppose that $f_{1}, \ldots, f_{n}$ are polyhedral proper concave functions on $\mathbb{R}$ with bounded effective domains. Then $\operatorname{cap}\left\{f_{1}, \ldots, f_{n}\right\}$ is also a polyhedral proper concave function with bounded effective domain, such that for any $x$ in the effective domain of $\operatorname{cap}\left\{f_{1}, \ldots, f_{n}\right\}$

$$
\operatorname{cap}\left\{f_{1}, \ldots, f_{n}\right\}(x)=\max \sum_{i=1}^{n} \lambda_{i} f_{i}\left(x_{i}\right),
$$

where the maximum is taken over all $\lambda_{1}, \ldots, \lambda_{n} \geq 0$ and $x_{1} \in \operatorname{dom} f_{1}, \ldots, x_{n} \in$ $\operatorname{dom} f_{n}$ satisfying

$$
\sum_{i=1}^{n} \lambda_{i}=1, \quad \sum_{i=1}^{n} \lambda_{i} x_{i}=x .
$$

Remark 2.1 Carathéodory's theorem allows a stronger assertion, namely that the maximum in 2.2 is attained for some $\lambda_{1}, \ldots, \lambda_{n}$ and $x_{1}, \ldots, x_{n}$ as above such that all except at most two of the $\lambda_{i}$ 's are zero. 
Proof outline of Lemma 2.2 The lemma follows from results in Rockafellar [Roc97. The representation for cap $\left\{f_{1}, \ldots, f_{n}\right\}$ is a direct consequence of Theorem 5.6 in combination with Corollary 9.8.3. Corollary 17.2.1 and Theorem 7.1 ensure that $\operatorname{cap}\left\{f_{1}, \ldots, f_{n}\right\}$ is polyhedral.

Let $f$ be a proper concave function. For any $x \in \operatorname{dom} f$ we denote by $D^{-} f(x)$ and $D^{+} f(x)$ the left and right derivatives of $f$ at $x$, adopting the convention that

$$
\begin{array}{ll}
D^{-} f(x)=+\infty & \text { if } f(y)=-\infty \text { for all } y<x \\
D^{+} f(x)=-\infty & \text { if } f(y)=-\infty \text { for all } y>x
\end{array}
$$

Since $f$ is concave, for each $x \in \operatorname{dom} f$

$$
D^{-} f(x) \geq D^{+} f(x) .
$$

\section{Option Prices}

We shall consider a European option to be exercised at time $T$ by the delivery of a portfolio $(\xi, \zeta)$ of cash and stock. Here $\xi$ and $\zeta$ are $\mathcal{F}_{T}$-measurable random variables. The portfolio $(\xi, \zeta)$ will be referred to as the option payoff.

Definition 3.1 The ask price (upper hedging price) and the bid price (lower hedging price) of a European option with payoff $(\xi, \zeta)$ and exercise time $T$ are defined, respectively, by

$$
\begin{aligned}
& \pi^{\mathrm{a}}(\xi, \zeta)=\min \left\{\alpha_{0} \mid(\alpha, \beta) \in \Phi\left(S^{\mathrm{a}}, S^{\mathrm{b}}\right), \vartheta_{T}\left(\alpha_{T}-\xi, \beta_{T}-\zeta\right) \geq 0\right\}, \\
& \pi^{\mathrm{b}}(\xi, \zeta)=\max \left\{-\alpha_{0} \mid(\alpha, \beta) \in \Phi\left(S^{\mathrm{a}}, S^{\mathrm{b}}\right), \vartheta_{T}\left(\alpha_{T}+\xi, \beta_{T}+\zeta\right) \geq 0\right\} .
\end{aligned}
$$

The minimum and maximum are attained because the corresponding sets are closed and, respectively, bounded below and above in the discrete setting. Any strategy realising the minimum or maximum will be called an optimal hedging strategy for the writer and, respectively, for the buyer of the option.

The inequality $\vartheta_{T}\left(\alpha_{T}-\xi, \beta_{T}-\zeta\right) \geq 0$ in Definition 3.1 is a superreplication condition, ensuring that the option writer who follows the self-financing strategy $(\alpha, \beta)$ and delivers the payoff $(\xi, \zeta)$ at time $T$ will end up holding a solvent portfolio $\left(\alpha_{T}-\xi, \beta_{T}-\zeta\right)$. Similarly, $\vartheta_{T}\left(\alpha_{T}+\xi, \beta_{T}+\zeta\right) \geq 0$ means that the option buyer who follows strategy $(\alpha, \beta)$ will end up holding a solvent portfolio $\left(\alpha_{T}+\xi, \beta_{T}+\zeta\right)$, having received the option payoff $(\xi, \zeta)$.

It is enough to develop algorithms to compute the ask price and writer's optimal hedging strategy for an arbitrary European option. This is because the bid and ask option prices are connected by

$$
\pi^{\mathrm{b}}(\xi, \zeta)=-\pi^{\mathrm{a}}(-\xi,-\zeta),
$$

and the optimal hedging strategy for the buyer of an option with payoff $(-\xi,-\zeta)$ is the same as the optimal hedging strategy for the writer of an option with payoff $(\xi, \zeta)$. 


\section{Ask Price Algorithm}

Algorithm 4.1 For a European option exercised by the delivery of a portfolio $(\xi, \zeta)$ of cash and stock at time $T$ we construct adapted processes $Z_{t}$ and $\tilde{Z}_{t}$ for $t=0,1, \ldots, T$ with values among polyhedral proper concave functions with bounded domain as follows:

- We put

$$
Z_{T}^{x}=\tilde{Z}_{T}^{x}= \begin{cases}\xi+x \zeta & \text { if } x \in\left[S_{T}^{\mathrm{b}}, S_{T}^{\mathrm{a}}\right], \\ -\infty & \text { if } x \notin\left[S_{T}^{\mathrm{b}}, S_{T}^{\mathrm{a}}\right] .\end{cases}
$$

This defines a function $x \mapsto Z_{T}^{x}(\mu)$ at each node $\mu \in \Omega_{T}$.

- For any $t=0,1, \ldots, T-1$ we take

$$
\tilde{Z}_{t}(\mu)=\operatorname{cap}\left\{Z_{t+1}(\nu) \mid \nu \in \Omega_{t+1} \text { is a successor node of } \mu\right\},
$$

at each node $\mu \in \Omega_{t}$, and put

$$
Z_{t}^{x}= \begin{cases}\tilde{Z}_{t}^{x} & \text { if } x \in\left[S_{t}^{\mathrm{b}}, S_{t}^{\mathrm{a}}\right] \\ -\infty & \text { if } x \notin\left[S_{t}^{\mathrm{b}}, S_{t}^{\mathrm{a}}\right]\end{cases}
$$

- The algorithm returns

$$
\max _{x \in \mathbb{R}} Z_{0}^{x},
$$

shown in Theorem 4.2 to be the ask price $\pi^{\mathrm{a}}(\xi, \zeta)$ of the option.

Observe that the lack of arbitrage guarantees that each function $Z_{t}(\mu)$ for $t=0,1, \ldots, T$ and $\mu \in \Omega_{t}$ takes at least one finite value. In particular, this means that the algorithm returns a finite value $\max _{x \in \mathbb{R}} Z_{0}^{x}$. This is so because $\mathcal{P} \neq \emptyset$ by Theorem 2.1, and for any $(P, S) \in \mathcal{P}$ we clearly have $Z_{t}^{S_{t}}>-\infty$ for each $t=0,1, \ldots, T$.

This algorithm can readily be implemented on a computer. We present some numerical examples in Section 5 . Observe that each of the polyhedral proper concave functions featuring in the algorithm can be uniquely characterised by the finite set of the extreme points of its endograph. This provides a natural way of encoding such functions in computer memory. Computing the concave cap is then a straightforward discrete optimisation procedure, as is evaluating the maximum in the final step.

\subsection{Hedging Strategy}

Lemma 4.1 Let $t=0,1, \ldots, T-1$ and let $\gamma, \delta$ be $\mathcal{F}_{t}$-measurable random variables such that for each $x \in \mathbb{R}$

$$
\gamma+x \delta \geq Z_{t}^{x}
$$

Then there are $\mathcal{F}_{t}$-measurable random variables $\rho, \sigma$ such that for each $x \in \mathbb{R}$

$$
\rho+x \sigma \geq Z_{t+1}^{x}
$$


and

$$
\vartheta_{t}(\gamma-\rho, \delta-\sigma) \geq 0
$$

Proof Let $y$ be an $\mathcal{F}_{t}$-measurable random variable such that

$$
Z_{t}^{y}-y \delta=\max \left\{Z_{t}^{x}-x \delta \mid x \in \mathbb{R}\right\} .
$$

Because $Z_{t}$ is a polyhedral proper concave function with bounded domain, the maximum is attained. In particular, it follows that

$$
y \in\left[S_{t}^{\mathrm{b}}, S_{t}^{\mathrm{a}}\right], \quad \tilde{Z}_{t}^{y}=Z_{t}^{y}>-\infty .
$$

If we can find $\sigma$ and $\rho$ such that 4.3 holds and

$$
D^{-} \tilde{Z}_{t}^{y} \geq \sigma \geq D^{+} \tilde{Z}_{t}^{y}, \quad \rho+y \sigma=\tilde{Z}_{t}^{y},
$$

then it will follow by the concavity and the construction of $\tilde{Z}_{t}$ that for any $x \in \mathbb{R}$

$$
\rho+x \sigma \geq \tilde{Z}_{t}^{x} \geq Z_{t+1}^{x}
$$

proving (4.2).

To prove that we can indeed find such $\sigma$ and $\rho$, we consider the following four cases, covering all possibilities:

1. $S_{t}^{\mathrm{b}}<y<S_{t}^{\mathrm{a}}$. Then $D^{-} Z_{t}^{y}=D^{-} \tilde{Z}_{t}^{y}, D^{+} Z_{t}^{y}=D^{+} \tilde{Z}_{t}^{y}$, and 4.4 implies that $D^{-} Z_{t}^{y} \geq \delta \geq D^{+} Z_{t}^{y}$. We put

$$
\sigma=\delta, \quad \rho=Z_{t}^{y}-y \sigma .
$$

As a result, 4.5 holds. Observe that

$$
\begin{aligned}
\rho+y \sigma & =Z_{t}^{y} \leq \gamma+y \delta, \\
\sigma & =\delta,
\end{aligned}
$$

which implies 4.3 .

2. $S_{t}^{\mathrm{b}}=y<S_{t}^{\mathrm{a}}$. Then $D^{+} Z_{t}^{y}=D^{+} \tilde{Z}_{t}^{y}$ and 4.4 implies that $\delta \geq D^{+} Z_{t}^{y}$.

We put

$$
\sigma=\min \left\{\delta, D^{-} \tilde{Z}_{t}^{y}\right\}, \quad \rho=Z_{t}^{y}-y \sigma .
$$

As a result, 4.5 holds. Moreover,

$$
\begin{aligned}
\rho+y \sigma & =Z_{t}^{y} \leq \gamma+y \delta, \\
\sigma & \leq \delta .
\end{aligned}
$$

Since $y=S_{t}^{\mathrm{b}}$, this implies 4.3.

3. $S_{t}^{\mathrm{b}}<y=S_{t}^{\mathrm{a}}$. In this case we put

$$
\sigma=\max \left\{\delta, D^{+} \tilde{Z}_{t}^{y}\right\}, \quad \rho=Z_{t}^{y}-y \sigma,
$$

and follow a similar argument as in case 2 . 
4. $S_{t}^{\mathrm{b}}=y=S_{t}^{\mathrm{a}}$. We take any finite $\sigma$ such that

$$
D^{-} \tilde{Z}_{t}^{y} \geq \sigma \geq D^{+} \tilde{Z}_{t}^{y}
$$

and put

$$
\rho=Z_{t}^{y}-y \sigma .
$$

Clearly, 4.5 holds. It also follows that

$$
\rho+y \sigma=Z_{t}^{y} \leq \gamma+y \delta,
$$

and since $S_{t}^{\mathrm{b}}=y=S_{t}^{\mathrm{a}}, 4.3$ is satisfied.

This completes the proof of Lemma 4.1 .

Observe that this proof of Lemma 4.1 provides concrete formulae for $\sigma$ and $\delta$. We can therefore construct a strategy $(\alpha, \beta)$ hedging the position of the writer of a European option with payoff $(\xi, \zeta)$, as in the following algorithm.

Algorithm 4.2 We construct a self financing strategy $(\alpha, \beta)$ by induction as follows:

- Put

$$
\alpha_{0}=\max _{x \in \mathbb{R}} Z_{0}^{x}, \quad \beta_{0}=0 .
$$

Then $\alpha_{0}, \beta_{0}$ are $\mathcal{F}_{0}$-measurable and for each $x \in \mathbb{R}$

$$
\alpha_{0}+x \beta_{0} \geq Z_{0}^{x} .
$$

- For any $t=1, \ldots, T$, if $\mathcal{F}_{t-1}$-measurable random variables $\alpha_{t-1}, \beta_{t-1}$ such that for each $x \in \mathbb{R}$

$$
\alpha_{t-1}+x \beta_{t-1} \geq Z_{t-1}^{x}
$$

have already been constructed, then Lemma 4.1 provides $\mathcal{F}_{t-1}$-measurable random variables $\alpha_{t}, \beta_{t}$ such that for each $x \in \mathbb{R}$

$$
\alpha_{t}+x \beta_{t} \geq Z_{t}^{x}
$$

and

$$
\vartheta_{t}\left(\alpha_{t-1}-\alpha_{t}, \beta_{t-1}-\beta_{t}\right) \geq 0 .
$$

Since $\alpha_{t}, \beta_{t}$ are $\mathcal{F}_{t-1}$-measurable, they are also $\mathcal{F}_{t}$-measurable, which makes it possible to iterate the last step.

As a result, we obtain a strategy $(\alpha, \beta) \in \Phi\left(S^{\mathrm{a}}, S^{\mathrm{b}}\right)$ such that for each $x \in\left[S_{T}^{\mathrm{b}}, S_{T}^{\mathrm{a}}\right]$

implying that

$$
\alpha_{T}+x \beta_{T} \geq Z_{T}^{x}=\xi+x \zeta,
$$

$$
\vartheta_{T}\left(\alpha_{T}-\xi, \beta_{T}-\zeta\right) \geq 0 .
$$

By Definition 3.1 it follows that

$$
\pi^{\mathrm{a}}(\xi, \zeta) \leq \alpha_{0}=\max _{x \in \mathbb{R}} Z_{0}^{x} .
$$

In Theorem 4.2 we shall prove that $(\alpha, \beta)$ is in fact an optimal hedging strategy for the writer, that is, $\pi^{\mathrm{a}}(\xi, \zeta)=\alpha_{0}$. 


\subsection{Representations for Ask Price}

The theorem below establishes various representations for the ask price $\pi^{\mathrm{a}}(\xi, \zeta)$, namely in terms of the writer's hedging strategy, the pricing algorithm, and the expectations under (possibly degenerate) risk-neutral probabilities. The last representation in terms of non-degenerate (equivalent to $Q$ ) risk neutral probabilities is known, see Jouini and Kallal [JK95], but here it is obtained by a very different technique.

In particular, the theorem proves the correctness of Algorithms 4.1 and 4.2 for computing the option ask price and writer's optimal hedging strategy under proportional transaction costs.

Theorem 4.2 The ask price of a European option with payoff $(\xi, \zeta)$ and exercise time $T$ can be represented as

$$
\begin{aligned}
\pi^{\mathrm{a}}(\xi, \zeta) & =\alpha_{0} \\
& =\max _{x \in \mathbb{R}} Z_{0}^{x} \\
& =\max _{(P, S) \in \overline{\mathcal{P}}} \mathbb{E}_{P}\left(\xi_{T}+S_{T} \zeta_{T}\right) \\
& =\sup _{(P, S) \in \mathcal{P}} \mathbb{E}_{P}\left(\xi_{T}+S_{T} \zeta_{T}\right),
\end{aligned}
$$

where $\alpha_{0}$ is the initial value of the writer's hedging strategy $(\alpha, \beta)$ constructed in Algorithm 4.2, $Z_{0}$ is the polyhedral proper concave function constructed in Algorithm 4.1, the sets $\mathcal{P}$ and $\overline{\mathcal{P}}$ are defined in Section 2.1, and $\mathbb{E}_{P}$ denotes the expectation under probability measure $P$.

Proof By Lemma 2.2, for any $t=1, \ldots, T$, any node $\mu \in \Omega_{t-1}$ and any $x \in \operatorname{dom} Z_{t-1}(\mu)$ there are numbers $y(\nu) \in \operatorname{dom} Z_{t}(\nu)$ and $\lambda(\nu) \geq 0$ for each node $\nu \in \operatorname{succ} \mu$ such that

$$
\begin{aligned}
1 & =\sum_{\nu \in \operatorname{succ} \mu} \lambda(\nu), \\
x & =\sum_{\nu \in \operatorname{succ} \mu} \lambda(\nu) y(\nu), \\
Z_{t-1}^{x}(\mu) & =\sum_{\nu \in \operatorname{succ} \mu} \lambda(\nu) Z_{t}^{y(\nu)}(\nu) .
\end{aligned}
$$

Using this property, we can construct by induction adapted processes $\hat{S}, \hat{Z}$ and a probability measure $\hat{P}$ on $\Omega$ such that:

$$
\hat{Z}_{0}=\max _{x \in \mathbb{R}} Z_{0}^{x}=Z_{0}^{\hat{S}_{0}}
$$


- For each $t=1, \ldots, T$ and each $\mu \in \Omega_{t-1}$ there are numbers $\lambda(\nu) \geq 0$ for each node $\nu \in \operatorname{succ} \mu$ satisfying

$$
\begin{aligned}
\hat{P}(\nu \mid \mu) & =\lambda(\nu), \\
\hat{S}_{t-1}(\mu) & =\sum_{\nu \in \operatorname{succ} \mu} \lambda(\nu) \hat{S}_{t}(\nu), \\
\hat{Z}_{t-1}(\mu) & =\sum_{\nu \in \operatorname{succ} \mu} \lambda(\nu) \hat{Z}_{t}(\nu) ;
\end{aligned}
$$

- For each $t=0,1, \ldots, T$

$$
\begin{aligned}
& S_{t}^{\mathrm{b}} \leq \hat{S}_{t} \leq S_{t}^{\mathrm{a}}, \\
& \hat{Z}_{t}=Z_{t}^{\hat{S}_{t}} .
\end{aligned}
$$

Such processes $\hat{S}, \hat{Z}$ and probability measure $\hat{P}$ may not be unique, in which case we choose any $\hat{S}, \hat{Z}, \hat{P}$ satisfying the above conditions.

It follows that $\hat{S}$ and $\hat{Z}$ are martingales under $\hat{P}$. As a result, $(\hat{P}, \hat{S}) \in \overline{\mathcal{P}}$ and

$$
\mathbb{E}_{\hat{P}}\left(\xi+\hat{S}_{T} \zeta\right)=\mathbb{E}_{\hat{P}}\left(\hat{Z}_{T}\right)=\hat{Z}_{0}=\max _{x \in \mathbb{R}} Z_{0}^{x}
$$

This proves that

$$
\max _{x \in \mathbb{R}} Z_{0}^{x} \leq \max _{(P, S) \in \overline{\mathcal{P}}} \mathbb{E}_{P}\left(\xi+S_{T} \zeta\right) .
$$

Now, by Definition 3.1, we can take a strategy $(\gamma, \delta) \in \Phi\left(S^{\mathrm{a}}, S^{\mathrm{b}}\right)$ such that $\gamma_{0}=\pi^{\mathrm{a}}(\xi, \zeta)$ and $\vartheta_{T}\left(\gamma_{T}-\xi, \delta_{T}-\zeta\right) \geq 0$. Then $\xi+x \zeta \leq \gamma_{T}+x \delta_{T}$ for each $x \in\left[S_{T}^{\mathrm{b}}, S_{T}^{\mathrm{a}}\right]$. Also take an arbitrary pair $(P, S) \in \overline{\mathcal{P}}$. Because $S_{T}^{\mathrm{b}} \leq S_{T} \leq S_{T}^{\mathrm{a}}$, it follows that $\xi+S_{T} \zeta \leq \gamma_{T}+S_{T} \delta_{T}$. By Lemma 7.1 the process $\gamma+S \delta$ is a supermartingale under $P$. As a result,

$$
\mathbb{E}_{P}\left(\xi+S_{T} \zeta\right) \leq \mathbb{E}_{P}\left(\gamma_{T}+S_{T} \delta_{T}\right) \leq \gamma_{0}=\pi^{\mathrm{a}}(\xi, \zeta)
$$

for any $(P, S) \in \overline{\mathcal{P}}$. It follows that

$$
\max _{(P, S) \in \overline{\mathcal{P}}} \mathbb{E}_{P}\left(\xi+S_{T} \zeta\right) \leq \gamma_{0}=\pi^{\mathrm{a}}(\xi, \zeta) .
$$

Inequalities 4.7, (4.8) and (4.6) imply that

$$
\pi^{\mathrm{a}}(\xi, \zeta)=\alpha_{0}=\max _{x \in \mathbb{R}} Z_{0}^{x}=\max _{(P, S) \in \overline{\mathcal{P}}} \mathbb{E}_{P}\left(\xi+S_{T} \zeta\right) .
$$

To complete the proof it remains to observe that

$$
\max _{(P, S) \in \overline{\mathcal{P}}} \mathbb{E}_{P}\left(\xi+S_{T} \zeta\right)=\sup _{(P, S) \in \mathcal{P}} \mathbb{E}_{P}\left(\xi+S_{T} \zeta\right)
$$

by Lemma 7.2 .

Remark 4.1 By Remark 2.1 $\max _{(P, S) \in \overline{\mathcal{P}}} \mathbb{E}_{P}\left(\xi_{T}+S_{T} \zeta_{T}\right)$ is attained at some $(P, S) \in \overline{\mathcal{P}}$ such that $P$ is concentrated on a binomial subtree. 


\section{$5 \quad$ Numerical Results}

We can apply the results of this paper to price European options with various payoffs under transaction costs. This includes not only calls or puts with either cash settlement or physical delivery, but also option baskets, such as a straddle or a butterfly. Under transaction costs, option baskets are by no means equivalent to linear combinations of the constituent options, so the results are non-trivial, illustrating the flexibility of the pricing algorithm, which is indeed applicable to arbitrary payoffs.

The first two numerical examples are based on the binomial tree model. We also provide one involving a trinomial tree model under proportional transaction costs to illustrate the point that the algorithm can deal not only with arbitrary payoffs, but also with arbitrary discrete models, and is by no means limited to complete ones.

The binomial tree examples overlap with the numerical work in earlier papers on proportional transaction costs, in particular, Boyle and Vorst BV92, Edirsinghe, Naik and Uppal [ENU93, and Palmer [Pal01a]. In each case we extend the results to parameter ranges (particularly, to large transaction costs) not covered by these earlier papers, and also to cases theoretically covered, but for which the earlier computational procedures failed to produce satisfactory results due to lack of accuracy or excessive demand for computing time and resources. This serves to show that the method proposed in the present paper is not only general enough to cover all such cases, but also that it is computationally more efficient than the previous approaches. It can also be noted that the implementation of our algorithm is straightforward.

Example 5.1 We consider the binomial tree model as in Boyle and Vorst BV92 and Palmer Pal01a]. The stock price process satisfies

$$
S_{t}=\varepsilon_{t} S_{t-1}
$$

for $t=1,2, \ldots, T$ with $S_{0}>0$, where $\varepsilon_{1}, \varepsilon_{2}, \ldots$ is a sequence of independent identically distributed random variables that take only two values

$$
e^{-\sigma \sqrt{h}}, \quad e^{\sigma \sqrt{h}}
$$

both with positive probability, with $h=\frac{1}{T}, T$ being the number of time steps per annum, and with $\sigma>0$ the stock volatility per annum. We assume given an effective (annually compounded) interest rate $r_{\mathrm{e}}>0$ and a transaction cost rate $k \in[0,1)$. For each $t=1, \ldots, T$ the bid and ask stock prices are

$$
S_{t}^{\mathrm{b}}=(1-k) S_{t}, \quad S_{t}^{\mathrm{a}}=(1+k) S_{t} .
$$

To ensure consistency with BV92, we also assume that there are no transaction costs at time 0 , so that

$$
S_{0}^{\mathrm{b}}=S_{0}^{\mathrm{a}}=S_{0} .
$$

In Table 1 we present the ask and bid prices of a European call option with strike price $K$ exercised at time $T h=1$ year by the delivery of a portfolio

$$
\left(-K 1_{S_{T}>K}, 1_{S_{T}>K}\right)
$$


of cash and stock. The option prices, computed by means of Algorithm 4.1 for a range of parameter values of $T, K, k$, given the volatility $\sigma=20 \%$, effective rate $r_{\mathrm{e}}=10 \%$ and initial stock price $S_{0}=100$, can be compared with those in Boyle and Vorst BV92, Tables I and IV, and Palmer Pal01a, Table 2.1. Apart from a few self-evident typographical or rounding errors, marked by superscipts $a$ and $c$, the main differences can be seen in the bid option prices for $k=2 \%$ and $T=52$ or 250 , indicated by superscript $b$. In these cases the assumptions in Boyle and Vorst BV92 do not hold and their method provides no results, Palmer Pal01a gives the cost of a strictly replicating strategy, whereas here we compute the (negative of the) cost of the optimal superreplicating strategy for the call buyer.

Table 1 Bid and ask prices of European calls with physical delivery under transaction costs after Boyle and Vorst BV92 and Palmer Pal01a]

\begin{tabular}{|c|c|c|c|c|c|c|c|c|}
\hline \multirow{3}{*}{$\begin{array}{c}\text { Strike } \\
\text { Price } \\
(K)\end{array}$} & \multicolumn{8}{|c|}{ Number of time steps $(T)$} \\
\hline & 6 & 6 & 13 & 13 & 52 & 52 & 250 & 250 \\
\hline & bid & ask & bid & ask & bid & ask & bid & ask \\
\hline & \multicolumn{8}{|c|}{$k=0 \%$} \\
\hline 80 & 27.703 & 27.703 & 27.701 & 27.701 & 27.665 & 27.665 & 27.675 & 27.675 \\
\hline 90 & 19.821 & 19.821 & 19.740 & 19.740 & 19.667 & 19.667 & 19.674 & 19.674 \\
\hline 100 & 12.655 & 12.655 & 13.093 & 13.093 & 12.953 & 12.953 & 12.984 & 12.984 \\
\hline 110 & 8.129 & 8.129 & 8.026 & 8.026 & 7.972 & 7.792 & 7.965 & 7.965 \\
\hline \multirow[t]{2}{*}{120} & 4.216 & 4.216 & 4.427 & 4.427 & 4.548 & 4.548 & 4.551 & 4.551 \\
\hline & \multicolumn{8}{|c|}{$k=0.125 \%$} \\
\hline 80 & 27.671 & 27.735 & 27.656 & 27.747 & 27.582 & 27.753 & 27.502 & 27.876 \\
\hline 90 & 19.749 & 19.894 & 19.638 & 19.842 & 19.469 & 19.865 & 19.246 & 20.103 \\
\hline 100 & 12.538 & 12.770 & 12.935 & 13.248 & 12.637 & 13.256 & 12.286 & 13.630 \\
\hline 110 & 8.003 & 8.254 & 7.843 & 8.205 & 7.604 & 8.324 & 7.136 & 8.715 \\
\hline \multirow[t]{2}{*}{120} & 4.102 & 4.329 & 4.256 & 4.595 & 4.202 & 4.882 & 3.773 & 5.269 \\
\hline & \multicolumn{8}{|c|}{$k=0.5 \%$} \\
\hline 80 & 27.582 & 27.837 & 27.534 & 27.894 & 27.383 & 28.047 & 27.273 & 28.574 \\
\hline 90 & $19.531^{a}$ & 20.113 & 19.333 & 20.149 & 18.889 & 20.453 & 18.221 & 21.346 \\
\hline 100 & $12.168^{a}$ & 13.106 & 12.445 & 13.699 & 11.597 & 14.111 & 9.684 & 15.339 \\
\hline 110 & 7.614 & 8.618 & 7.269 & 8.721 & 6.374 & 9.300 & 3.647 & 10.649 \\
\hline \multirow[t]{2}{*}{120} & 3.754 & 4.663 & 3.726 & 5.084 & 3.077 & 5.820 & 0.879 & 7.161 \\
\hline & \multicolumn{8}{|c|}{$k=2 \%$} \\
\hline 80 & 27.327 & 28.297 & 27.276 & 28.563 & $27.273^{b}$ & 29.409 & $26.683^{b}$ & 31.568 \\
\hline 90 & 18.697 & 20.983 & 18.281 & 21.346 & $17.521^{b}$ & 22.643 & $18.182^{b}$ & 25.524 \\
\hline 100 & 10.323 & 14.358 & 10.115 & 15.333 & $7.697^{b}$ & 16.966 & $7.485^{b}$ & 20.413 \\
\hline 110 & 5.845 & 9.965 & 4.311 & $10.554^{c}$ & $0.865^{b}$ & 12.469 & $-0.038^{b}$ & 16.192 \\
\hline 120 & 2.266 & 5.926 & 1.266 & 6.859 & $0.000^{b}$ & 8.950 & $-0.012^{b}$ & 12.750 \\
\hline
\end{tabular}

${ }^{a}$ Agrees with Pal01a, but differs from BV92.

${ }^{b}$ The cost of the optimal superreplicating strategy differs from that of the strictly replicating strategy in $\mathrm{Pal01a}$; the method in BV92 does not apply here.

${ }^{c}$ Differs from [BV92]. 
Remark 5.1 The two negative bid option prices in Table 1 mean that a long position in the option can only be hedged upon receiving a positive amount of cash. This is due to the fact that the physical delivery method with payoff $\left(-K 1_{S_{T}>K}, 1_{S_{T}>K}\right)$ forces the holder to exercise the option at nodes where $(1-k) S_{T}<K<S_{T}$, when it may not necessarily be beneficial to exercise. This anomaly is discussed and resolved in Perrakis and Lefoll PL04 in the context of American puts, but their argument is also applicable to European call and put options.

Example 5.2 Next we apply our pricing algorithm to options with various payoffs, namely a call, a bear spread and a butterfly, and compare the numerical results with those in Edirsinghe, Naik and Uppal ENU93. The computations are based on a binomial model with transaction costs similar to that in Example 5.1. except that we follow ENU93 in assuming that transaction costs are absent not only at time 0 but also at the option expiry time,

$$
S_{T}^{\mathrm{b}}=S_{T}^{\mathrm{a}}=S_{T} .
$$

We can therefore restrict our attention without loss of generality to options with cash delivery. The call option will have strike price 100 . The bull spread can be regarded as a basket consisting of a long call with strike price 97.5 and a short call with strike price 102.5. The butterfly is a basket of one long call with strike price 97.5 and one with strike price 102.5, and two short calls with strike price 100 . We take volatility $\sigma=10 \%$, effective rate $r_{\mathrm{e}}=0 \%$, and the time to expiry to be one year for each of the options.

Table 2 Ask prices of European options with various payoffs under transaction costs compared with Edirsinghe, Naik and Uppal ENU93.

\begin{tabular}{c|ccccc}
\hline Transaction & \multicolumn{5}{|c}{ Number of time steps $(T)$} \\
cost rate $(k)$ & 8 & 19 & 52 & 250 & 1000 \\
\hline & \multicolumn{5}{|c}{ Call } \\
$0.0 \%$ & 3.865 & $4.040^{a}$ & $3.969^{c}$ & $3.984^{c}$ & $3.987^{c}$ \\
$2.5 \%$ & $6.092^{d}$ & $7.130^{a}$ & $8.535^{c}$ & $11.861^{c}$ & $16.244^{c}$ \\
$5.0 \%$ & 7.736 & $9.240^{a}$ & $11.389^{c}$ & $16.247^{c}$ & $22.486^{c}$ \\
\hline \multicolumn{5}{|c}{ Bull spread } \\
$0.0 \%$ & 2.403 & $2.409^{a}$ & $2.401^{c}$ & $2.402^{c}$ & $2.403^{c}$ \\
$2.5 \%$ & 3.270 & $4.273^{b}$ & $4.425^{c}$ & $4.602^{c}$ & $4.690^{c}$ \\
$5.0 \%$ & 3.567 & $4.370^{a}$ & $4.541^{c}$ & $4.688^{c}$ & $4.756^{c}$ \\
\hline \multicolumn{5}{|c}{ Butterfly } \\
$0.0 \%$ & 0.683 & $0.072^{a}$ & $0.275^{c}$ & $0.249^{c}$ & $0.249^{c}$ \\
$2.5 \%$ & 2.134 & $0.212^{a}$ & $2.500^{c}$ & $2.500^{c}$ & $2.500^{c}$ \\
$5.0 \%$ & 2.500 & $0.216^{a}$ & $2.500^{c}$ & $2.500^{c}$ & $2.500^{c}$ \\
\hline
\end{tabular}

\footnotetext{
${ }^{a}$ Computed in ENU93 by an approximate algorithm, but not by an exact algorithm.

${ }^{b}$ Unable to compute in ENU93.

${ }^{c}$ Not covered in ENU93.

${ }^{d}$ Differs from the value in ENU93.
} 
The results are presented in Table 2 for a range of values of the transaction cost rate $k$ and the number of time steps $T$. With two exceptions, one most likely a transcription mistake and one option price which could not be obtained by the algorithms in [ENU93, they agree to within a rounding or approximation error with the values in ENU93 computed by an exact linear programming algorithm for $T=8$ and an approximate algorithm for $T=19$. We also present results computed with the aid of Algorithm 4.1 for larger values of $T$ not covered in ENU93, namely $T=52,250$ and 1000 time steps.

Example 5.3 Consider the trinomial model such that for $t=1,2, \ldots, T$ the stock price process satisfies

$$
S_{t}=\varepsilon_{t} S_{t-1}
$$

with $S_{0}>0$, where $\varepsilon_{1}, \varepsilon_{2}, \ldots$ is a sequence of independent identically distributed random variables that take only three possible values

$$
e^{-\sigma \sqrt{h}}, \quad 1, \quad e^{-\sigma \sqrt{h}}
$$

each with positive probability, where $h=\frac{1}{T}, T$ being the number of time steps per annum, and where $\sigma>0$ is the stock volatility. Also given is the effective (annually compounded) interest rate $r_{\mathrm{e}}>0$ and transaction cost rate $k \in[0,1)$. The bid and ask stock prices are

$$
S_{t}^{\mathrm{b}}=(1-k) S_{t}, \quad S_{t}^{\mathrm{a}}=(1+k) S_{t}
$$

for each $t=0,1, \ldots, T$.

In Table 3 we give the bid and ask prices of a call option and a bull spread with cash settlement and one year to expiry, computed by Algorithm 4.1 for a range of values of $k$ and $T$. The call payoff is $\left(S_{T}-K\right) 1_{S_{T}>K}$ with strike price $K=100$. The bull spread is a basket consisting of one long call with strike price $K=95$ and one short call with strike price $K=105$. We take $S_{0}=100$,

\begin{tabular}{|c|c|c|c|c|c|c|}
\hline \multirow{3}{*}{$\begin{array}{c}\text { Transaction } \\
\text { cost rate } \\
(k)\end{array}$} & \multicolumn{6}{|c|}{ Number of time steps $(T)$} \\
\hline & 12 & 12 & 52 & 52 & 250 & 250 \\
\hline & ask & bid & ask & bid & ask & bid \\
\hline & \multicolumn{6}{|c|}{ Call } \\
\hline $0 \%$ & 12.822 & 9.091 & 12.953 & 9.091 & 12.984 & 9.091 \\
\hline $1 \%$ & 15.330 & 7.111 & 16.515 & 7.111 & 18.622 & 7.111 \\
\hline $2 \%$ & 17.751 & 5.169 & 19.699 & 5.169 & 23.141 & 5.169 \\
\hline $3 \%$ & 20.102 & 3.266 & 22.681 & 3.266 & 27.163 & 3.266 \\
\hline & \multicolumn{6}{|c|}{ Bull spread } \\
\hline $0 \%$ & 8.398 & 4.897 & 8.764 & 4.823 & 9.007 & 4.688 \\
\hline $1 \%$ & 8.918 & 3.668 & 9.091 & 3.106 & 9.091 & 2.385 \\
\hline $2 \%$ & 9.090 & 2.705 & 9.091 & 2.120 & 9.091 & 1.531 \\
\hline $3 \%$ & 9.091 & 1.965 & 9.091 & 1.496 & 9.091 & 1.029 \\
\hline
\end{tabular}
$r_{\mathrm{e}}=10 \%$ and $\sigma=20 \%$.

Table 3 Bid and ask prices of options in the trinomial model 


\section{Concluding Remarks}

In this paper we have established algorithms for computing the ask and bid prices and optimal superreplicating strategies for European options under proportional transaction costs on the underlying asset. The results apply to options with general payoffs, exercised by the delivery of a portfolio of cash and stock, in any discrete market model, and with no restrictions on the model or the transaction costs other than the lack of arbitrage. This general setting covers the earlier partial results by various authors, referred to in the introduction.

We put forward a dynamic programming procedure, in which the local optimisation problem at each tree node amounts to computing the concave cap of certain functions. This offers an elegant geometric picture behind the pricing and hedging algorithms, and hence has the potential to facilitate further generalisations.

For brevity and simplicity we assume no friction on the risk free security and only a single underlying stock. Nevertheless, the algorithms and results can be extended in a straightforward manner to different deposit and credit interest rates, as well as several underlying stocks, each with its own bid-ask spread. An extension to American options is also possible, and will be reported in forthcoming work.

Numerical examples are presented here which have been produced by means of the pricing algorithm. These extend existing numerical work in various directions simultaneously: much wider parameter ranges, arbitrary option payoffs, including option baskets, a wider range of models with transaction costs, including the standard binomial and trinomial approximations to the Black-Scholes model, and, importantly, no restrictions on the magnitude of transaction costs.

\section{Appendix}

Here we give two technical lemmas used in the paper.

Lemma 7.1 If $(\alpha, \beta) \in \Phi\left(S^{\mathrm{a}}, S^{\mathrm{b}}\right)$ and $(P, S) \in \overline{\mathcal{P}}$, then $\alpha+\beta S$ is a supermartingale under $P$.

Proof Since $S_{t}^{\mathrm{b}} \leq S_{t} \leq S_{t}^{\mathrm{a}}$, the self-financing condition 2.1] implies that

$$
\alpha_{t}-\alpha_{t+1} \geq\left(\beta_{t}-\beta_{t+1}\right)^{-} S_{t}^{\mathrm{a}}-\left(\beta_{t}-\beta_{t+1}\right)^{+} S_{t}^{\mathrm{b}} \geq-\left(\beta_{t}-\beta_{t+1}\right) S_{t} .
$$

As a result, for each $t=0, \ldots, T-1$

$$
\mathbb{E}_{P}\left(\alpha_{t+1}+\beta_{t+1} S_{t+1} \mid \mathcal{F}_{t}\right)=\alpha_{t+1}+\beta_{t+1} S_{t} \leq \alpha_{t}+\beta_{t} S_{t},
$$

where $\mathbb{E}_{P}$ denotes the expectation under $P$.

Lemma 7.2 For any option payoff $(\xi, \zeta)$, any $(\bar{P}, \bar{S}) \in \overline{\mathcal{P}}$ and any $\delta>0$ there exists a pair $\left(P^{\delta}, S^{\delta}\right) \in \mathcal{P}$ such that

$$
\left|\mathbb{E}_{\bar{P}}\left(\xi+\zeta \bar{S}_{T}\right)-\mathbb{E}_{P^{\delta}}\left(\xi+\zeta S_{T}^{\delta}\right)\right|<\delta .
$$


Proof Due to the lack of arbitrage, there exists some $(P, S) \in \mathcal{P}$. If

$$
\mathbb{E}_{\bar{P}}\left(\xi+\zeta \bar{S}_{T}\right)=\mathbb{E}_{P}\left(\xi+\zeta S_{T}\right),
$$

then the result is trivial. Assume that this is not the case, and choose

$$
\varepsilon \in\left(0, \min \left\{1, \frac{\delta}{\left|\mathbb{E}_{\bar{P}}\left(\xi+\zeta \bar{S}_{T}\right)-\mathbb{E}_{\mathbb{P}}\left(\xi+\zeta S_{T}\right)\right|}\right\}\right) .
$$

If we now define

$$
P^{\delta}=(1-\varepsilon) \bar{P}+\varepsilon P,
$$

then $P^{\delta}$ is a probability measure equivalent to $Q$. Also let

$$
S_{t}^{\delta}=\mathbb{E}_{P^{\delta}}\left((1-\varepsilon) \bar{S}_{T} \frac{d \bar{P}}{d P^{\delta}}+\varepsilon S_{T} \frac{d P}{d P^{\delta}} \mid \mathcal{F}_{t}\right)
$$

for $t=0,1, \ldots, T$, which is clearly a martingale under $P^{\delta}$. It follows that

$$
\begin{aligned}
\mathbb{E}_{P^{\delta}}\left(\xi+\zeta S_{T}^{\delta}\right) & =\mathbb{E}_{P^{\delta}}(\xi)+\mathbb{E}_{P^{\delta}}\left(\zeta S_{T}^{\delta}\right) \\
& =(1-\varepsilon) \mathbb{E}_{\bar{P}}(\xi)+\varepsilon \mathbb{E}_{P}(\xi)+(1-\varepsilon) \mathbb{E}_{\bar{P}}\left(\zeta \bar{S}_{T}\right)+\varepsilon \mathbb{E}_{P}\left(\zeta S_{T}\right) \\
& =(1-\varepsilon) \mathbb{E}_{\bar{P}}\left(\xi+\zeta \bar{S}_{T}\right)+\varepsilon \mathbb{E}_{P}\left(\xi+\zeta \bar{S}_{T}\right) .
\end{aligned}
$$

As a result,

$$
\left|\mathbb{E}_{\bar{P}}\left(\xi+\zeta \bar{S}_{T}\right)-\mathbb{E}_{P^{\delta}}\left(\xi+\zeta S_{T}^{\delta}\right)\right|=\varepsilon\left|\mathbb{E}_{\bar{P}}\left(\xi+\zeta \bar{S}_{T}\right)-\mathbb{E}_{P}\left(\xi+\zeta S_{T}\right)\right|<\delta .
$$

To show that $\left(P^{\delta}, S^{\delta}\right) \in \mathcal{P}$ it remains to verify the inequalities $S_{t}^{\mathrm{b}} \leq S_{t}^{\delta} \leq S_{t}^{\mathrm{a}}$ for each $t=0,1, \ldots, T$. Take any such $t$ and any $\mathcal{F}_{t}$-measurable random variable $\eta \geq 0$. Then

$$
\begin{aligned}
\mathbb{E}_{P^{\delta}}\left(S_{t}^{\delta} \eta\right) & =\mathbb{E}_{P^{\delta}}\left(S_{T}^{\delta} \eta\right) \\
& =(1-\varepsilon) \mathbb{E}_{\bar{P}}\left(\bar{S}_{T} \eta\right)+\varepsilon \mathbb{E}_{P}\left(S_{T} \eta\right) \\
& =(1-\varepsilon) \mathbb{E}_{\bar{P}}\left(\bar{S}_{t} \eta\right)+\varepsilon \mathbb{E}_{P}\left(S_{t} \eta\right) \\
& \leq(1-\varepsilon) \mathbb{E}_{\bar{P}}\left(S_{t}^{\mathrm{a}} \eta\right)+\varepsilon \mathbb{E}_{P}\left(S_{t}^{\mathrm{a}} \eta\right) \\
& =\mathbb{E}_{P^{\delta}}\left(S_{t}^{\mathrm{a}} \eta\right) .
\end{aligned}
$$

This proves that $S_{t}^{\delta} \leq S_{t}^{\mathrm{a}}$. In a similar way we can show that $S_{t}^{\mathrm{b}} \leq S_{t}^{\delta}$, completing the proof.

\section{References}

[BLPS92] B. Bensaid, J. Lesne, H. Pagès, and J. Scheinkman, Derivative asset pricing with transaction costs, Math. Finance 2 (1992), 63-86.

[BV92] P. Boyle and T. Vorst, Option replication in discrete time with transaction costs, J. Finance 47 (1992), 271-293. 
[CP02] G.M. Constantinides and S. Perrakis, Stochastic dominance bounds on derivatives prices in a multiperiod economy with proportional transaction costs, J. Econom. Dynam. Control 26 (2002), 1323-1352.

[CPS05] G.-Y. Chen, K. Palmer, and Y.-C. Sheu, The least cost super replicating portfolio for short puts and calls in the Boyle-Vorst model with transaction costs, preprint, 2005.

[CZ99] G.M. Constantinides and T. Zariphopoulou, Bounds on prices of contingent claims in an intertemporal economy with proportional transaction costs and general preferences, Finance Stoch. 3 (1999), 345-369.

[CZ01] _ Bounds on derivative prices in an intertemporal setting with proportional transaction costs and multiple securities, Math. Finance 11 (2001), 331-346.

[DPZ93] M.H.A. Davis, V.G. Panas, and T. Zariphopoulou, European option pricing with transaction costs, SIAM J. Control Optim. 31 (1993), 470-493.

[DR91] J.C. Dermody and R.T. Rockafellar, Cash stream valuation in the face of transaction costs and taxes, Math. Finance 1 (1991), 31-54.

[ENU93] C. Edirsinghe, V. Naik, and R. Uppal, Optimal replication of options with transactions costs and trading restrictions, J. Fin. Quant. Anal. 28 (1993), 117-138.

[HN89] S.D. Hodges and A. Neuberger, Optimal replication of contingent claims under transaction costs, Rev. Futures Markets 8 (1989), 222239 .

[JK95] E. Jouini and H. Kallal, Martingales and arbitrage in securities markets with transaction costs, J. Econom. Theory 66 (1995), 178-197.

[Jou00] E. Jouini, Price functionals with bid-ask spreads: An axiomatic approach, J. Math. Econom. 34 (2000), 547-558.

[Koc04] M. Kociński, Hedging of the European option in discrete time under proportional transaction costs, Math. Methods Oper. Res. 59 (2004), $315-328$.

[KPT96] P.-F. Koehl, H. Pham, and N. Touzi, Option pricing under transaction costs: A martingale approach, Institut National de la Statistique et des Etudes Economiques, Working paper 9647, 1996.

[KPT99] _ Hedging in discrete time under transaction costs and continuous-time limit, J. Appl. Probab. 36 (1999), 163-178.

[KPT01] - On super-replication in discrete time under transaction costs, Theory Probab. Appl. 47 (2001), 667-673. 
[Kus95] S. Kusuoka, Limit theorem on option replication cost with transaction costs, Ann. Appl. Probab. 5 (1995), 198-221.

[LPS98] D. Lamberton, H. Pham, and M. Schweizer, Local risk-minimization under transaction costs, Mathematics of Operations Research 23 (1998), 585-612.

[Mer89] R.C. Merton, On the application of the continuous-time theory of finance to financial intermediation and insurance, The Geneva Papers on Risk and Insurance 14 (1989), 225-261.

[Mer90] , Continuous Time Finance, Blackwell, Oxford, 1990.

[Mon03] M. Monoyios, Effcient option pricing with transaction costs, J. Comput. Finance 7 (2003), 107-128.

[Mon04]__ Option pricing with transaction costs using a Markov chain approximation, J. Economic Dynamics \& Control 28 (2004), 889-913.

[MV97] F. Mercurio and T.C.F. Vorst, Options pricing and hedging in discrete time with transaction costs, Mathematics of Derivative Securities (M.A.H. Dempster and S.R. Pliska, eds.), Cambridge University Press, Cambridge, UK, 1997, pp. 190-215.

[Ort01] F. Ortu, Arbitrage, linear programming and martingales in securities markets with bid-ask spreads, Decis. Econom. Finance 24 (2001), no. 2, 79-105.

[Pal01a] K. Palmer, A note on the Boyle-Vorst discrete-time option pricing model with transaction costs, Math. Finance 11 (2001), 357-363.

[Pal01b] _ Replicating and super-replicating portfolios in the BoyleVorst discrete-time option pricing model with transaction costs, Working paper, University of Melbourne, 2001.

[PL97] S. Perrakis and J. Lefoll, Derivative asset pricing with transaction costs: an extension, Comput. Econom. 10 (1997), 359-376.

[PL04] _ The American put under transaction costs, J. Econom. Dynam. Control 28 (2004), 915-935.

[Roc97] R.T. Rockafellar, Convex analysis, Princeton University Press, Princeton, 1997.

[Rut98] M. Rutkowski, Optimality of replication in the CRR model with transaction costs, Appl. Math. (Warsaw) 25 (1998), 29-53.

[Ste97] E. Stettner, Option pricing in the CRR model with proportional transaction costs: A cone transformation approach, Appl. Math. (Warsaw) 24 (1997), 475-514. 
[Ste00] _ Option pricing in discrete-time incomplete market models, Math. Finance 10 (2000), 305-321.

[Tok04] K. Tokarz, European and American option pricing under proportional transaction costs, Ph.D. thesis, University of Hull, 2004.

[Tou99] N. Touzi, Super-replication under proportional transaction costs: From discrete to continuous-time models, Math. Meth. Oper. Res. 50 (1999), 297-320. 